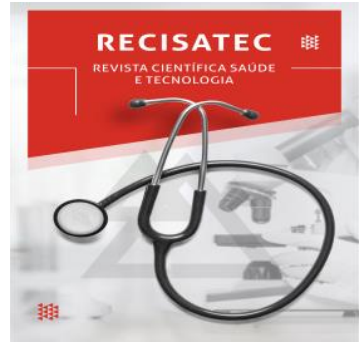

\title{
COMORBIDADES E CO-INFECÇÃO COMO FATORES DE RISCO ASSOCIADOS AO AGRAVAMENTO DE INFECÇÃO PELA COVID-19 EM PACIENTES ATENDIDOS NO CENTRO DE SAÚDE DE METANGULA, NO NORTE DE MOÇAMBIQUE
}

\author{
COMORBIDITIES AND COINFECTION AS RISK FACTORS ASSOCIATED WITH AGGRAVATION \\ INFECTION BY COVID-19 IN PATIENTS ATTENDED AT THE METANGULA HEALTH CENTER, IN \\ NORTHERN MOZAMBIQUE
}

\section{COMORBILIDADES Y COINFECCIÓN COMO FACTORES DE RIESGO ASOCIADOS AL AGRAVAMIENTO DE INFECCIÓN POR COVID-19 EN PACIENTES ATENDIDOS EN EL CENTRO DE SALUD METANGULA, NORTE DE MOZAMBIQUE}

Wilson Samuel ${ }^{1}$, Nércia Eugénio Monjane²

Submetido em: 12/08/2021

e1226

Aprovado em: 22/09/2021

https://doi.org/10.53612/recisatec.v1i2.26

\begin{abstract}
RESUMO
Objectivo: O presente estudo pretende avaliar os fatores de risco preexistentes associados ao agravamento de infecção pela COVID-19 em pacientes atendidos no Centro de Saúde de Metangula. Método: Estudo transversal e retrospectivo realizado a partir de dados secundários extraídos do boletim epidemiológico do Centro de Saúde de Metangula num período de 01 (um) ano. Foram obtidos os dados demográficos, condição clinica e resultado laboratorial da COVID-19. Os dados foram analisados e apresentados em forma de gráficos e tabela com números absolutos e relativos. Resultados: Os dados revelaram que ocorreram incidências de infecção por COVID-19 em todas as faixas etárias, com predominância na faixa etária de 30-39 anos e predominância de caso da COVID19 nos homens em comparação com mulheres. As comorbidades como diabetes mellitus, doenças respiratórias crónicas e os pacientes infectados com vírus da imunodeficiência humana (HIV), foram fatores de alto risco para o agravamento de infecção por COVID-19 entre os pacientes atendidos no Centro de Saúde de Metangula. Conclusão: Este estudo pode facilitar os prestadores de cuidados de saúde na identificação de pacientes com fatores de risco e, consequentemente, a diminuição no número de casos e de óbitos por essa doença. Os achados serão úteis para os tomadores de decisão na implementação de protocolos de manejos de pacientes com complicação gerados por determinada comorbidade e co-infecção quando da sua associação à infecção por coronavírus.
\end{abstract}

PALAVRAS-CHAVE: Comorbidades. Co-infecção. Fatores de risco. COVID-19. SARS-CoV-2.

\section{ABSTRACT}

Objective: This study aims to evaluate preexisting risk factors associated with aggravation of COVID19 infection in patients treated at the Metangula Health Center. Method: Cross-sectional and retrospective study based on secondary data extracted from the epidemiological bulletin of the Health Center of Metangula over a period of 01 (one) year. Demographic data, clinical condition and laboratory results from COVID-19 were obtained. Data were analyzed and presented in the form of graphs and tables with absolute and relative numbers. Results: The data revealed that incidences of COVID-19 infection occurred in all age groups, with a predominance in the age group of 30-39 years and a predominance of cases of COVID-19 in men compared to women. Comorbidities such as diabetes mellitus, chronic respiratory diseases and patients infected with human immunodeficiency virus (HIV) were high risk factors for the worsening of COVID-19 infection among patients treated at the Metangula Health Center. Conclusion: This study can facilitate health care providers in identifying patients with risk factors and, consequently, a decrease in the number of cases and deaths from this disease. The findings will be useful for decision makers in the implementation of management

\footnotetext{
1 Biólogo, especializado em Saúde - Departamento de Saúde Militar, MDN, Moçambique. Email de correspondência: wilsonito2011@gmail.com

2 Médica - Departamento de Saúde Militar, MDN, Moçambique
} 


\section{RECISATEC - REVISTA CIENTÍFICA SAÚDE E TECNOLOGIA ISSN 2763-8405}

COMORBIDADES E CO-INFECĈ̃O COMO FATORES DE RISCO ASSOCIADOS AO AGRAVAMENTO DE INFECĈ̃O PELA COVID-19 EM PACIENTES ATENDIDOS NO CENTRO DE SAÚDE DE METANGULA, NO NORTE DE MOÇAMBIQUE Wilson Samuel, Nércia Eugénio Monjane

protocols for patients with complications generated by certain comorbidities and coinfection when associated with coronavirus infection.

KEYWORDS: Comorbidities. Coinfection. Risk factors. COVID-19. SARS-CoV-2.

\section{RESUMEN}

Objetivo: Este estudio tiene como objetivo evaluar los factores de riesgo preexistentes asociados al agravamiento de la infección por COVID-19 en pacientes atendidos en el Centro de Salud de Metangula. Método: Estudio transversal y retrospectivo basado en datos secundarios extraídos del boletín epidemiológico del Centro de Salud de Metangula durante un período de 01 (un) año. Se obtuvieron datos demográficos, condición clínica y resultados de laboratorio de COVID-19. Los datos fueron analizados y presentados en forma de gráficos y tablas con números absolutos y relativos. Resultados: Los datos revelaron que las incidencias de la infección por COVID-19 ocurrieron en todos los grupos de edad, con predominio en el grupo de edad de 30 a 39 años y predominio de casos de COVID-19 en hombres frente a mujeres. Las comorbilidades como la diabetes mellitus, las enfermedades respiratorias crónicas y los pacientes infectados por el virus de la inmunodeficiencia humana (VIH) fueron factores de alto riesgo para el agravamiento de la infección por COVID-19 entre los pacientes atendidos en el Centro de Salud de Metangula. Conclusión: Este estudio puede facilitar a los proveedores de salud en la identificación de pacientes con factores de riesgo y, en consecuencia, una disminución en el número de casos y muertes por esta enfermedad. Los hallazgos serán de utilidad para los tomadores de decisiones en la implementación de protocolos de manejo para pacientes con complicaciones generadas por ciertas comorbilidades y coinfección cuando se asocian con la infección por coronavirus.

PALABRAS-CLAVE: Comorbilidades. Coinfección. Factores de riesgo. COVID-19. SARS-CoV-2.

\section{INTRODUÇÃO}

Em 31 de Dezembro de 2019, um surto de pneumonia causado por um novo coronavírus (SARS-CoV-2) foi relatado na cidade de Wuhan, China [1]. Desde então, a doença do coronavírus 19 (COVID-19) se espalhou rapidamente por diferentes continentes com um grande número de pessoas sendo infectadas em um curto período de tempo, desafiando a capacidade e os recursos do sistema de saúde em todo o planeta, que em 11 de Março de 2020, a Organização Mundial da Saúde ou (OMS) declarou COVID-19 como uma pandemia [1,2].

Sob a perspectiva moçambicana, a infecção por COVID-19 foi declarada pelo Ministério da Saúde como emergência de saúde pública de âmbito Nacional no mês de Março de 2020, e teve seu primeiro caso confirmado em Moçambique, no dia 22 de Março. Como medida de apoio ao Sistema de Saúde, foram implementadas as medidas de contenção, permitindo retardar o pico da pandemia da COVID-19 [3].

Analisando os dados clínicos e epidemiológicos da COVID-19 da última semana em Moçambique sugerem que as comorbidades específicas aumentam o risco de infecção com pior lesão pulmonar e morte. Além disso, uma alta proporção de pacientes com COVID-19 e outras condições sugerem comorbidades e co-infecção como um factor de risco potencial para pacientes com COVID-19 [1,3,23].

Fatores de risco dizem respeito a quaisquer condições que prejudiquem a resposta imunitária do organismo frente a uma enfermidade. Estudos sugerem que pacientes infectados com COVID-19 


\section{RECISATEC - REVISTA CIENTÍFICA SAÚDE E TECNOLOGIA ISSN 2763-8405}

COMORBIDADES E CO-INFECĈ̃O COMO FATORES DE RISCO ASSOCIADOS AO AGRAVAMENTO DE INFECÇÃO PELA COVID-19 EM PACIENTES ATENDIDOS NO CENTRO DE SAÚDE DE METANGULA, NO NORTE DE MOÇAMBIQUE Wilson Samuel, Nércia Eugénio Monjane

que possuam comorbidades apresentam maior vulnerabilidade imunológica bem como maior taxa de letalidade quando comparados à pacientes infectados somente pelo vírus. Entre as principais comorbidades listadas, incluem-se diabetes, doenças respiratórias crónicas e doenças cardiovasculares e co-infecção como Vírus da imunodeficiência humana $(\mathrm{HIV})$, pois têm maior risco de agravar a infecção pelo COVID-19 [4,5,23,24].

O mecanismo subjacente do SARS-CoV-2 permanece indefinido; entretanto, está estabelecido que o vírus usa receptores ACE-2, que se encontram na superfície das células hospedeiras para entrar na célula [5]. Citocinas pró-inflamatórias de alto plasma, linfopenia e manifestações respiratórias atípicas são os atributos de COVID-19. Várias doenças metabólicas e infecciosas afectam a gravidade de COVID-19 e desempenham um papel fundamental no estabelecimento de sintomas complexos. As comorbidades e co-infecção levam o paciente com COVID-19 a um círculo vicioso infeccioso e estão substancialmente associados à morbidade e mortalidade significativas [5,6,7].

Frente ao cenário mundial e diante do situação de COVID-19 em Moçambique, torna-se imprescindível realizar estudo a fim que se estabeleçam manejos adequados aos pacientes acometidos por doenças subjacentes ou condições clinicas que representem potenciais fatores de risco ao prognóstico de pacientes diagnósticados com SARS-CoV-2, visando atenuar as complicações apresentadas por eles [4].

\section{MÉTODOS E MATERIAL}

Trata-se de um estudo descritivo, transversal e retrospectivo que recorreu à recolha e análise dos dados secundários disponíveis nos livros de registos e boletim epidemiológica da COVID-19 no Centro de Saúde de Metangula (CSM), Distrito de Lago, província de Niassa, nestes livros de registos foram recolhido os seguintes dados; demográficos, condição clinica do paciente e resultado laboratorial da COVID-19 que foram confirmados através de análise molecular (RT PCR). Os dados recolhidos e analisados foram de todos os pacientes que realizaram o teste de diagnóstico da COVID-19, e que foram atendidos no CSM de forma consecutiva num período de 01 (um) ano (01 de Maio de 2020 a 31 de Maio de 2021) e teve os seguintes critérios: Critério de inclusão dos pacientes todos os pacientes que realizaram o teste de diagnóstico da COVID-19 e com dados demográficos, clínicos e laboratoriais completos; Critério de exclusão dos pacientes - todos os pacientes que não realizaram o teste de diagnóstico da COVID-19 e com dados demográficos, clínicos e laboratoriais incompletos.

Toda informação recolhida no âmbito deste estudo foi introduzida numa base de dados criada especificamente para o efeito. A análise dos dados foi descritiva, por um lado, e analítica por outro. A análise descritiva incluiu a apresentação da distribuição por género, a descrição dos grupos etários mais afectados pelo problema em estudo. Os resultados descritivos foram apresentados em forma de gráficos de frequência. Na parte analítica, procurou-se estabelecer associações entre variáveis em 


\section{RECISATEC - REVISTA CIENTÍFICA SAÚDE E TECNOLOGIA ISSN 2763-8405}

COMORBIDADES E CO-INFECÇÃO COMO FATORES DE RISCO ASSOCIADOS AO AGRAVAMENTO DE INFECÇÃO PELA COVID-19 EM PACIENTES ATENDIDOS NO CENTRO DE SAÚDE DE METANGULA, NO NORTE DE MOÇAMBQUE Wilson Samuel, Nércia Eugénio Monjane

estudo como: comorbidades, co-infecção e a infecção pelo COVID-19. Testes estatísticos (Chiquadrado, Odds Ratio) foram efectuados procurando uns níveis de significância de $95 \%(p<0,05)$. Foi criada uma base de dados num pacote estatístico SPSS (versão 24.0) o qual também foi utilizado para a análise efetuada no âmbito deste estudo, onde foram introduzidas as informações recolhidas.

\section{RESULTADOS}

Dos 503 casos suspeitos analisados neste estudo, atendido no Centro de Saúde de Metangula entre 01 de Maio de 2020 a 31 de Maio de 2021, 17,3\% (87) pacientes foram confirmados positivo, através de análise molecular (RT PCR), com COVID-19. No gráfico 1, estão sumariadas a distribuição de casos da COVID-19 por sexo. Já relacionado ao sexo, houve uma predominância do sexo masculino nos casos confirmados $54(10,7 \%)$ quando comparado ao sexo feminino $33(6,6 \%)$.

\section{Grafico.1. Distribuição de casos da COVID-19 por sexo}

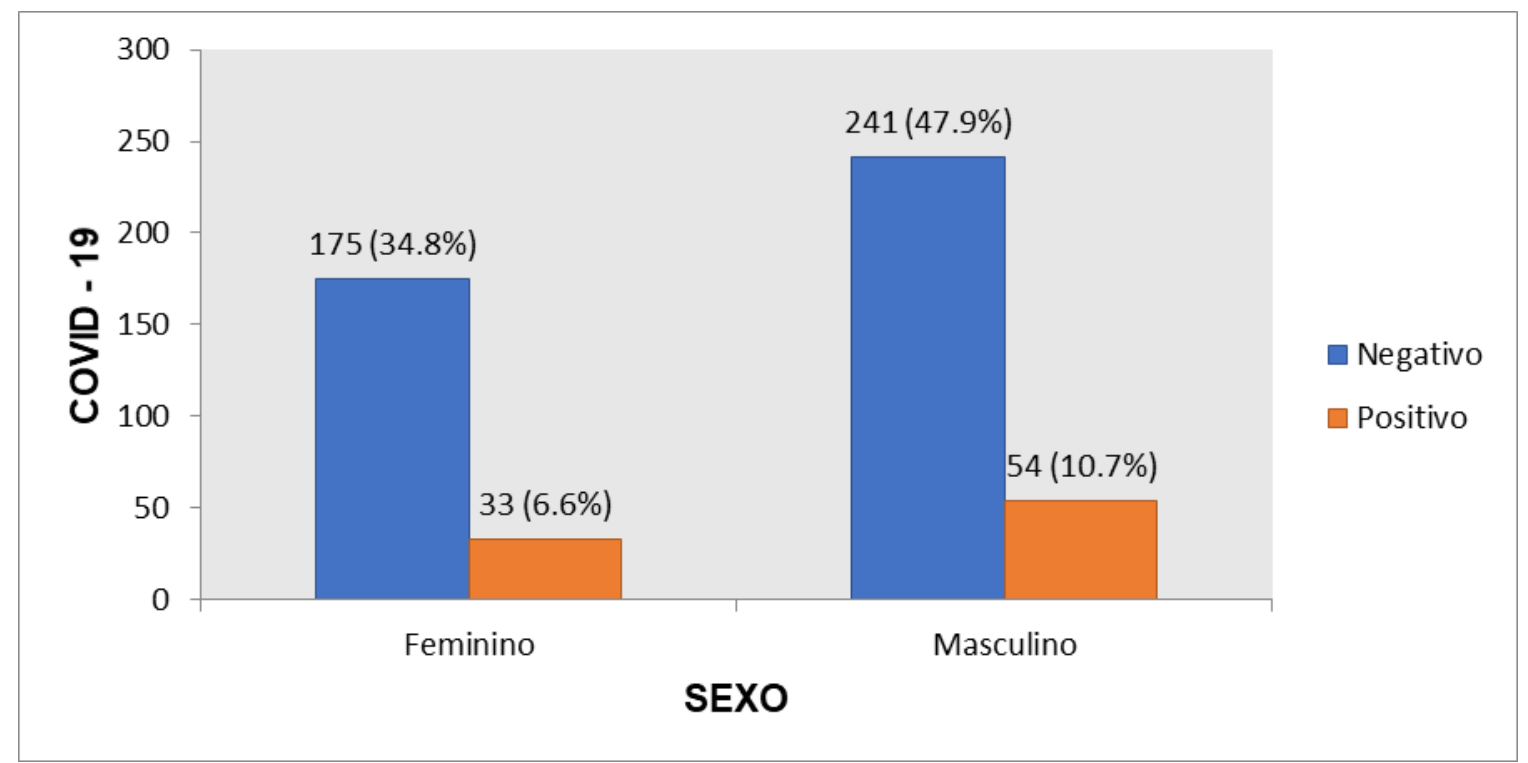

Fonte: Dados da pesquisa, 2021.

Em relação ao perfil dos pacientes com casos confirmados de COVID-19, houve uma predominância na faixa etária de 30-39 anos, sendo $5,4 \%$ dos casos positivo quando comparado a outras faixas etárias. (Gráfico. 2). 
COMORBIDADES E CO-INFECÇÃO COMO FATORES DE RISCO ASSOCIADOS AO AGRAVAMENTO DE INFECĈ̃O PELA COVID-19 EM PACIENTES ATENDIDOS NO CENTRO DE SAÚDE DE METANGULA, NO NORTE DE MOÇAMBIQUE Wilson Samuel, Nércia Eugénio Monjane

\section{Grafico.2. Distribuição de casos da COVID-19 segundo faixa etária.}

Fonte: Dados da pesquisa, 2021

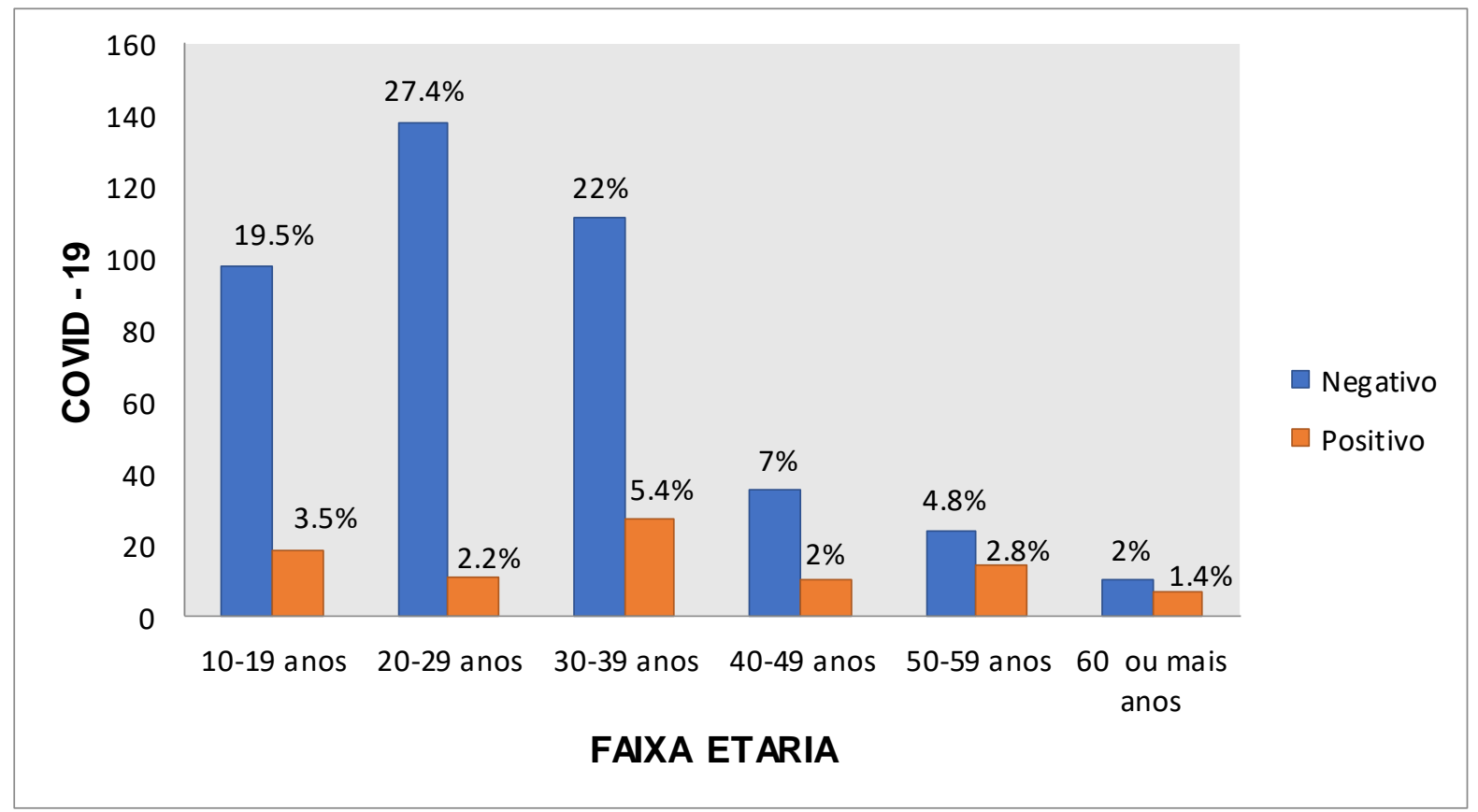

As comorbidades e co-infecção associados ao agravamento da infecção pela COVID-19 em pacientes atendidos no centro de saúde de Metangula estão sumariados na Tabela 1. A proporção maior de infecção por COVID-19 foi para pacientes infectados com vírus da imunodeficiência humana (HIV) com 31 (6,2\%); seguido das doenças respiratórias crónicas com cerca de 30 (6\%) e Diabetes Mellitus (DM) com cerca de 24 (4,8\%). Em relação as comorbidades e co-infecção associadas à COVID-19, os pacientes infectados com HIV eram estatisticamente mais frequentemente propensos à COVID-19 (OR = 12.844, IC 95\% = 5.245 -31.454), o mesmo aconteceu aos pacientes com Diabetes Mellitus (OR $=12.121$, IC 95\% = (3.601- 40.794) e os pacientes que apresentaram as doenças respiratórias crónicas $(\mathrm{OR}=7.236$, IC 95\% = 3,036 -17,247). Cerca de 8 (1.6\%) apresentavam as doenças cardiovascular e $3(0.6 \%)$ a Hepatite B. Porém, não se encontrou nenhuma associação estatística entre essas variáveis e ao agravamento de infecção por COVID-19 p> 0.05. 
COMORBIDADES E CO-INFECCÃO COMO FATORES DE RISCO ASSOCIADOS AO AGRAVAMENTO DE INFECÇ̃̃O PELA COVID-19 EM PACIENTES ATENDIDOS NO CENTRO DE SAÚDE DE METANGULA, NO NORTE DE MOÇAMBIQUE Wilson Samuel, Nércia Eugénio Monjane

Tabela.1 Comorbidades e co-infecção preexistentes associados ao agravamento de infecção pela COVID-19 em pacientes atendidos no Centro de Saúde de Metangula

\begin{tabular}{|c|c|c|c|c|}
\hline COMORBIDADES E CO-INFECÇÃO & POSITIVO & NEGATIVO & OR (IC95\%) & VALOR DE P \\
\hline & $n=87(17.3 \%)$ & $n=416(82.7 \%)$ & & \\
\hline $\begin{array}{l}\text { Diabetes Mellitus } \\
\text { Sim }\end{array}$ & $24(4.8 \%)$ & $6(1.2 \%)$ & & \\
\hline Não & $63(12.5 \%)$ & $410(81.5 \%)$ & $12.121(3.601-40.794)$ & 0.001 \\
\hline $\begin{array}{l}\text { Doenças respiratórias crónicas } \\
\text { Sim }\end{array}$ & $30(6 \%)$ & $12(2.4 \%)$ & & \\
\hline Não & $57(11.3 \%)$ & $404(80.3 \%)$ & $7.236(3.036-17.247)$ & 0.001 \\
\hline $\begin{array}{l}\text { Doenças cardiovascular } \\
\text { Sim }\end{array}$ & $8(1.6 \%)$ & $10(2.0 \%)$ & & \\
\hline Não & $79(15.7 \%)$ & $406(80.7 \%)$ & $0.481(0.084-2.752)$ & 0.411 \\
\hline $\begin{array}{l}\text { HIV+ } \\
\text { Sim }\end{array}$ & $31(6.2 \%)$ & $9(1.8 \%)$ & & \\
\hline Não & $56(11.1 \%)$ & $407(80.9 \%)$ & $12.844(5.245-31.454)$ & 0.001 \\
\hline $\begin{array}{l}\text { Hepatite B } \\
\text { Sim }\end{array}$ & $3(0.6 \%)$ & $3(0.6 \%)$ & & \\
\hline Não & $84(16.7 \%)$ & $413(82.1 \%)$ & $0.612(0.175-9.278)$ & 0.612 \\
\hline
\end{tabular}

Fonte: Dados da pesquisa, 2021

\section{DISCUSSÃO}

Neste estudo, houve uma predominância de caso da COVID-19 no sexo masculino com 54 $(10,7 \%)$ em comparação com os $33(6,6 \%)$ restantes do sexo feminino, os dados estão de acordo com estudos recentes [9], e em contraste com alguns outros estudos que mostraram que as mulheres eram predominantemente infectadas por SARS-CoV-2 [10,13]. A alta susceptibilidade entre os homens no Município de Metangula pode estar relacionados ao facto de que os homens terem a obrigação de sustentar suas famílias, portanto constituíam a maioria da população ativa em empregos, como pesca, comércio, construção, logística, e outros serviços essenciais durante o surto da COVID-19, o que aumentou seus contactos e interacções na sociedade. Pelo contrário, a maioria das atividades desempenhadas por mulheres permitiu-lhes permanecer em casa e realizar os trabalhos domésticos. Além disso, a população masculina mais jovem tem maiores tendências de se envolver em atividades mais sociais, esportivas e culturais que podem envolver grandes encontros. Por outro lado, estudos recentes ligaram a baixa susceptibilidade em mulheres a variações na imunidade inata, homónios esteróides e outros fatores associados aos cromossomos sexuais [12]. 


\section{RECISATEC - REVISTA CIENTÍFICA SAÚDE E TECNOLOGIA ISSN 2763-8405}

COMORBIDADES E CO-INFECÇÃO COMO FATORES DE RISCO ASSOCIADOS AO AGRAVAMENTO DE INFECÇÃO PELA COVID-19 EM PACIENTES ATENDIDOS NO CENTRO DE SAÚDE DE METANGULA, NO NORTE DE MOÇAMBIQUE Wilson Samuel, Nércia Eugénio Monjane

Por exemplo, níveis reduzidos de testosterona têm sido amplamente associados à alta gravidade em homens e podem ser responsáveis por resultados favoráveis em mulheres infectadas com COVID-19 [13].

A faixa etária, com maior número de casos suspeitos notificados no presente estudo foi de 30-39 anos, com 5,4\% dos casos, os dados estão de acordo com estudos realizado no Brasil com 27,1\% dos casos, na faixa etária de 30-39 anos [14] e por outro lado, estudos realizados nos países africanos, mostram que a COVID -19 afeta as pessoas mais jovens, entretanto, isso se deve ao perfil demográfico dos países africanos por ter maior taxa de natalidade que resulta na maior índice da população jovem em relação à população idosa e por outro lado essa predominância do intervalo de 30-39 anos pode ser explicada por essa faixa etária que está inclusa no intervalo de idade que mais procura serviços de saúde em Moçambique (18-40 anos), e também pela possível relação entre a procura pelo serviço de saúde e a produtividade económica laboral. Estes resultados, no entanto, contradizem alguns estudos anteriores que sugeriram idades acima de 50 e 60 anos como fatores de alto risco [15].

No que refere à associação entre pacientes com diabete mellitus (DM) e o quadro da COVID19 observado neste estudo, está de acordo com os estudos recentes [4,5,14]. Conforme afirmado anteriormente, o DM aumenta as complicações da COVID-19 e o risco de mortalidade relacionada ao COVID-19 [1, 4,14]. Evidências atuais demonstram que pacientes com DM têm maior probabilidade de apresentar sintomas e complicações graves do que pacientes sem DM devido ao COVID-19 [16]. Uma hipótese é que a hiperglicemia facilita a entrada do vírus nas células, uma vez que a ACE2 e o vírus precisam de glicose para sua função $[17,18]$. Do outro lado, há possibilidade do vírus piorar as condições clínicas do paciente, tendo em vista que o SARS-COV-2 promove o aumento da glicemia em pacientes com diabetes tipo 2 [18]. O agravamento no quadro de diabéticos pode estar relacionado com o fato de essa comorbidade diminuir a imunidade natural do organismo [17].

Neste estudo, a preexistência de doenças respiratórias crónicas estão associados a infecção por COVID-19, o achado é compatível com os dados de estudo meta-análise que investigou as doenças respiratórias crónicas relacionados com COVID-19 [19]. Sabe-se que o sistema imunológico responde à infecção pelo SARS-CoV-2 com um processo inflamatório marcado pela ação de células imunitárias, necrose tecidual e hiperplasia local, efeitos estes que podem prejudicar o processo de troca gasosa dos pulmões e gerar um quadro de pneumonia grave [20], o que pode justificar o motivo das doenças respiratórias serem um fator de risco para o COVID-19, outra possibilidade deve se a maior expressão dos receptores da enzima conversora da angiotensina 2 em pacientes com doenças respiratórias crónicas, facilitando a entrada do vírus da SARS-CoV-2 na célula [20].

Apesar das doenças cardiovascular não serem associados à infecção por COVID-19 neste estudo, sabe-se que tais comorbidades pioram o prognóstico em pacientes com COVID-19 e podem determinar o excedente de mortes, especialmente quando estão envolvidos diversos fatores [21]. 


\section{RECISATEC - REVISTA CIENTÍFICA SAÚDE E TECNOLOGIA ISSN 2763-8405}

COMORBIDADES E CO-INFECÇÃO COMO FATORES DE RISCO ASSOCIADOS AO AGRAVAMENTO DE INFECÇÃO PELA COVID-19 EM PACIENTES ATENDIDOS NO CENTRO DE SAÚDE DE METANGULA, NO NORTE DE MOÇAMBIQUE Wilson Samuel, Nércia Eugénio Monjane

Algumas condições de comprometimento imunológico podem contribuir para susceptibilidade as infecções por COVID-19 e sua gravidade, como os pacientes infectados com vírus da imunodeficiência humana (HIV). De acordo com os dados do presente estudo observou-se que o HIV foi associado à um risco significativamente maior de infecção por COVID-19, o achado é compatível com os dados de estudo de meta-análise que descobriu que pessoas vivendo com HIV têm um risco maior de infecção por SARS-CoV-2 e risco de mortalidade por COVID-19 do que pessoas sem HIV [22]. Diante do pressuposto sugere-se que os pacientes com COVID-19 associado à HIV, têm altos níveis de citocinas pró-inflamatórias e quimiocinas levando à um aumento na gravidade das infecções por COVID-19, maior consumo de células T CD4 + e CD8 +, diminuição das células T regulatórias e ambiente imune inato levando a uma tempestade de citocinas e piora do tecido danificado [23,24]. Além disso, as evidências também sugerem que subgrupos de pacientes com COVID-19 podem sofrer de síndrome hiperinflamatória denominada linfo-histiocitose hemofagocítica secundária, que é frequentemente desencadeada por infecções virais subjacentes ou sepse e leva à hipercitocinemia fulminante e fatal com falência de múltiplos órgãos [23]. Embora a incidência de linfo-histiocitose hemofagocítica secundária entre pacientes com HIV possa ser rara, a co-infecção de pacientes com HIV com COVID-19 pode resultar em linfo-histiocitose hemofagocítica secundária levando a maior gravidade da doença e alta mortalidade [23].

\section{CONCLUSÃO}

Os resultados revelaram que ocorreram incidências de infecção por COVID-19 em todas as faixas etárias, com predominância na faixa etária de 30-39 anos e predominância de caso de COVID19 nos homens em comparação com as mulheres. Algumas comorbidades relatadas, como doenças cardiovasculares e os pacientes infectados com vírus da Hepatite B, não foram estatisticamente significativa com agravamento de infecção por COVID-19 entre os pacientes. Pelo contrário as comorbidades como diabetes Mellitus, doenças respiratórias crónicas e os pacientes infectados com vírus da imunodeficiência humana (HIV), foram fatores de alto risco para agravamento de infecção por COVID-19 entre os pacientes atendidos no Centro de Saúde de Metangula. Este estudo pode facilitar os prestadores de cuidados de saúde na identificação de pacientes com fatores de risco e, consequentemente, diminuição no número de casos e de óbitos por essa doença. Os achados serão úteis para os tomadores de decisão na implementação de protocolos de manejos de pacientes com complicações gerados por determinada comorbidade e co-infecção aquando da sua associação à infecção por coronavírus. 


\section{RECISATEC - REVISTA CIENTÍFICA SAÚDE E TECNOLOGIA ISSN 2763-8405}

COMORBIDADES E CO-INFECCÃO COMO FATORES DE RISCO ASSOCIADOS AO AGRAVAMENTO DE INFECĈ̃O PELA COVID-19 EM PACIENTES ATENDIDOS NO CENTRO DE SAÚDE DE METANGULA, NO NORTE DE MOÇAMBIQUE Wilson Samuel, Nércia Eugénio Monjane

\section{REFERÊNCIAS BIBLIOGRÁFICAS}

1. Renu Kaviyarasi et al. "Coronaviruses pathogenesis, comorbidities and multi-organ damage A review." Life sciences. 2020;255:17839. doi: https://doi.org/10.1016/..Ifs.2020.117839

2. Samuel Wilson et al. Papel da biossegurança em laboratórios de análises clínicas no cenário da pandemia do novo Coronavírus (SARS-CoV-2). Revista Científica Multidisciplinar Núcleo do Conhecimento. 2021 jun;06(04):108-123. Doi: 10.32749/nucleodoconhecimento.com.br/saude/cenario-da-pandemia

3. Organização Mundial Da Saúde (OMS). Caso da COVID-19 confirmado em Moçambique. 2020. [Acesso em: 2021 ago 20] Disponível em:

https://www.afro.who.int/pt/news/organizacao-mundial-da-saude-caso-da-covid-19confirmado-em-mocambique

4. Singh AK et al. "Diabetes in COVID-19: Prevalence, pathophysiology, prognosis and practical considerations." Diabetes \& metabolic syndrome. 2020;14(4):303-310. doi: https://doi.org/10.1016/i.dsx.2020.04.004

5. Yang $\mathrm{J}$ et al. "Prevalence of comorbidities and its effects in patients infected with SARS-CoV2: a systematic review and meta-analysis." International journal of infectious diseases: IJID: official publication of the International Society for Infectious Diseases. 2020;94:91-95. doi: https://doi.org/10.1016/i.ijid.2020.03.017

6. Li Wenhui et al. "Angiotensin-converting enzyme 2 is a functional receptor for the SARS coronavirus." Nature. 2003;426(6965):450-4. doi: https://doi.org/10.1038/nature02145

7. Li Yan-Chao et al. "The neuroinvasive potential of SARS-CoV2 may play a role in the respiratory failure of COVID-19 patients." Journal of medical virology. 2020;92(6):552-555. doi: https://doi.org/10.1002/jmv.25728

8. Rothan HA, Siddappa NB. "The epidemiology and pathogenesis of coronavirus disease (COVID-19) outbreak." Journal of autoimmunity. 2020;109:102433. doi: https://doi.org/10.1016/j.jaut.2020.102433

9. Ortolan A et al. "Does gender influence clinical expression and disease outcomes in COVID19 ? A systematic review and meta-analysis." International journal of infectious diseases: IJID: official publication of the International Society for Infectious Diseases. 2020;99:496-504. doi: https://doi.org/10.1016/i.ijid.2020.07.076

10. Yehia BR et al. "Association of Race With Mortality Among Patients Hospitalized With Coronavirus Disease 2019 (COVID-19) at 92 US Hospitals." JAMA network open. 2020 Aug;3(8):e2018039. doi: https://doi.org/10.1001/jamanetworkopen.2020.18039

11. Xie J et al. "Metabolic Syndrome and COVID-19 Mortality Among Adult Black Patients in New Orleans." Diabetes care. 2020 Aug;44(1):188-193. doi:10.2337/dc20-1714

12. Conti $P$, Younes A. Coronavirus cov-19/sars-cov- 2 affects women less than men: clinical response to viral infection. J Biol Regul Homeost Agents. 2020;34:339. doi: https://doi.org/10.23812/Editorial-Conti-3

13. Auerbach JM, Khera M. Testosterone's Role in COVID-19. J Sex Med. 2021;18(5):843-848. doi: https://doi.org/10.1016/i.jsxm.2021.03.004

14. Silva AWC. Et al. Perfil epidemiológico e determinante social do COVID-19 em Macapá, Amapá, Amazônia, Brasil. Revista Científica Multidisciplinar Núcleo do Conhecimento. 2020 Abr;05(04):05-27. DOI: https://doi.org/10.32749/nucleodoconhecimento.com.br/saude/covid19-em-macapa

15. Shen $Y$ et al. "Epidemiology and clinical course of COVID-19 in Shanghai, China." Emerging microbes \& infections. 2020;9(1):1537-1545. doi: https://doi.org/10.1080/22221751.2020.1787103

16. lacobellis G. "COVID-19 and diabetes: Can DPP4 inhibition play a role?." Diabetes research and clinical practice. 2020;162:108125. doi: https://doi.org/10.1016/..diabres.2020.108125

17. Ma RCW, Holt RIG. "COVID-19 and diabetes." Diabetic medicine: a journal of the British Diabetic Association. 2020;37(5):723-725. doi: https://doi.org/10.1111/dme.14300 


\section{RECISATEC - REVISTA CIENTÍFICA SAÚDE E TECNOLOGIA ISSN 2763-8405}

COMORBIDADES E CO-INFECCÃO COMO FATORES DE RISCO ASSOCIADOS AO AGRAVAMENTO DE INFECCÃO PELA COVID-19 EM PACIENTES ATENDIDOS NO CENTRO DE SAÚDE DE METANGULA, NO NORTE DE MOÇAMBIQUE Wilson Samuel, Nércia Eugénio Monjane

18. Bornstein SR et al. "Practical recommendations for the management of diabetes in patients with COVID-19." The lancet. Diabetes \& endocrinology. 2020;8(6):546-550. doi: https://doi.org/10.1016/S2213-8587(20)30152-2

19. Wang B et al. "Does comorbidity increase the risk of patients with COVID-19: evidence from meta-analysis." Aging. 2020;12(7): 6049-6057. doi: https://doi.org/10.18632/aging.103000

20. Martín-Giménez VM et al. "Lungs as target of COVID-19 infection: Protective common molecular mechanisms of vitamin $D$ and melatonin as a new potential synergistic treatment." Life sciences. 2020;254:117808. doi: https://doi.org/10.1016/..Ifs.2020.117808

21. Guan Wei-Jie et al. "Cardiovascular comorbidity and its impact on patients with COVID19." The European respiratory journal. 2020;55(6):2001227. doi: https://doi.org/10.1183/13993003.01227-2020

22. Ssentongo $P$ et al. "Epidemiology and outcomes of COVID-19 in HIV-infected individuals: a systematic review and meta-analysis." Scientific reports. 2021;11(1):6283. doi: https://doi.org/10.1038/s41598-021-85359-3

23. QIN, Chuan et al. "Dysregulation of Immune Response in Patients With Coronavirus 2019 (COVID-19) in Wuhan, China." Clinical infectious diseases: an official publication of the Infectious Diseases Society of America. 2020;71(15):762-768. doi: https://doi.org/10.1093/cid/ciaa248

24. Mehta $P$ et al. "COVID-19: consider cytokine storm syndromes and immunosuppression." Lancet (London, England). 2020;395(10229):1033-1034. doi: https://doi.org/10.1016/S0140-6736(20)30628-0 